\title{
Grand challenge: the emerging field of neurohospitalist medicine
}

\author{
James F. Meschia* \\ Mayo Clinic, Jacksonville, FL, USA \\ ${ }^{*}$ Correspondence: meschia.james@mayo.edu
}

Frontiers in Hospitalist Neurology (FHN) is a new peer-reviewed, open-access journal formed to respond to the growing momentum of the neurohospitalist movement. Cultivating a subspecialty peer-reviewed medical literature will be vital if neurohospitalist medicine is to flourish among more established medical subspecialties. Peer-review has a storied past. In the English speaking world, the process of scientific peer review is said to have originated in 1752 when the Royal Society of London officially assumed responsibility for the Philosophical Transactions (Kronick, 1990). Though peer review has its limitations, a better system has yet to be invented for evaluating and encouraging scientific progress. FHN has been established to provide a venue for peer-reviewed articles that address issues directly relevant to the care of patients with neurological abnormalities in the inpatient setting. It remains to be seen how intellectually vibrant neurohospitalist medicine will be, as it is defined more by the process of healthcare delivery than by a set of diseases with a common pathophysiology like epileptology or common symptoms like behavioral neurology. While numerous renowned neurology peer-reviewed journals exist and are receptive to high-impact original articles pertaining to diseases encountered in the inpatient setting, a subspecialty journal presently does not exist that would be receptive to publication of trial protocols and the results of early phase research devoted to management issues of direct relevance to neurohospitalists. $F H N$ will be receptive to high quality articles of registered trials with the expectation that this will improve and encourage the design and execution of more controlled trials.

The new field of neurohospitalist medicine continues to define its scope of practice. By definition, the primary responsibility of a neurohospitalist is to provide care to patients with neurological abnormalities that either lead to hospitalization or complicate hospital stay. Common inpatient conditions for which neurological expertise is often provided include delirium, either uncomplicated or superimposed on a chronic neurological condition like dementia or Parkinson's disease; acute ischemic and hemorrhagic stroke; poorly controlled epilepsy; CNS infections, including encephalitis, meningitis and epidural abscesses; sedative withdrawal syndromes; cranial and spinal trauma; other acute spinal cord compressive syndromes; and Status migrainosus. Clinical judgment is essential for the effective practice of hospitalist neurology. Maintaining the proper place for clinical judgment will require pushing back against opposing trends in medicine (Ropper, 2009). Clinical judgment may be lacking in such common clinical encounters as admission of patients with transient ischemic attack (Josephson et al., 2008). Validated clinical tools like the ABCD2 score for transient ischemic attack have the potential to rationalize and standardize care for common inpatient neurological conditions (Asimos et al., 2010). Neurohospitalists could be at the front lines of implementation of practice improvements.

The diversity and acuity of conditions that are the purview of neurohospitalists mean that training standards for practitioners must remain high. Every accredited neurology residency program is obligated to have inpatient training incorporated into the curriculum. However, recent and proposed future changes in resident hours, lengths of shifts, and mandatory continuity clinics may be eroding the inpatient training experience of neurology residents. The shift-worker culture may parallel postgraduate practice models, but it does not add to the ability of residents to learn from their experiences through even short-term inpatient follow-up. One solution to the dilution of clinical experience in the residency years is to have fellowship training focused solely on inpatient care. Academic fellowships in hospitalist neurology have been inaugurated at several medical centers, including the University of California San Francisco, the University of Washington in Seattle, and Mayo Clinic Florida. Frontiers could provide an opportunity for Program Directors to formally describe curricula, assessment tools, recruitment and retention experiences, and outcomes of trainees.

Population-based studies tend to focus on individual diseases like stroke. More reliable data on the utilization of neurological services in the inpatient setting are needed to fully appreciate current practices in both the academic and community settings. Some information concerning case mix of academic inpatient neurology services is gathered to prepare periodic reports of admissions and consultations for the American College of Graduate Medical Education. It might be possible for this data to be analyzed and summarized to gain insights into inpatient practices in the academic setting. Beyond this administrative data, the field might benefit from a prospective multicenter registry similar to the NINDS Stroke Data Bank, which gathered data on 1,806 patients from 4 academic centers from 1978 to 1986 (Foulkes et al., 1988). Care that is provided by accredited academic neurology programs, while vital, represents only a small proportion of the care delivered on a population level. It is not well known how divergent care is with regard to the evaluation and management of common inpatient neurological diagnoses.

Neurohospitalists should consider joining the checklist movement for improving patient care through better adherence to guidelines and improved standardization of process of care (Hales and Pronovost, 2006). The use of checklists for improving quality control has demonstrated dramatic effectiveness in specific inpatient settings, including the operating room, where a checklist intervention was shown to reduce deaths and surgical complications across multiple medical settings globally (Haynes 
et al., 2009). Close collaboration with general medical hospitalists and neurosurgeons would greatly expand the opportunities for developing checklists that reduce key errors in common clinical settings.

Medical errors are a significant cause of death. One decade ago, the Institute of Medicine estimated that 44,000 to 98,000 people die each year because of preventable medical errors (Kohn et al., 2000). Following the report, there has been a rapid rise in the number of patient safety publications and research awards (Stelfox et al., 2006). Progress in patient safety has been made since the call to action by the Institute of Medicine, but there is still a need to improve safety metrics (Wachter, 2010). Neurohospitalists could play a role in helping to ensure that safety metrics are applied and interpreted in an appropriate manner for patients with acute neurological injury.

Some safety metrics need to be tailored with an eye toward the neurologically impaired patient. The Centers for Medicare and Medicaid services considers catheterassociated urinary tract infections (UTI) to be a preventable condition. Patients with stroke are susceptible to UTI (Poisson et al., 2010). This will translate into higher rates of UTI on services with a high proportion of stroke patients. To the uninformed observer, this could appear as a sign that stroke patients are receiving poor quality care compared to other groups who are not as susceptible to UTI. The goal should be to prevent UTI emerging as a new condition among patients admitted for care of stroke, whether or not a catheter is involved.
Remove all catheters; eliminate all catheter-associated complications. However, this may come with its own unintended consequences, such as skin breakdown, more falls when transferring from bed to bathroom, or agitation associated with more episodes of bladder distention.

There is a tendency to define a new specialty by drawing bright lines between the new specialty and more traditional specialties. Nonetheless, it is likely that patients would benefit most by a team approach to patient management. This has been shown to be the case for neurointensivists. For example, co-management of subarachnoid hemorrhage by neurointensivists has been shown to improve outcomes (Josephson et al., 2010).Similar salutary effects may accrue from involvement of the neurohospitalist in the care of a diverse group of inpatients, though this remains to be proven.

The editorial staff at FHN looks forward to making a contribution to development of the field of hospitalist neurology.

\section{REFERENCES}

Asimos, A. W., Johnson, A. M., Rosamond, W. D., Price, M. F., Rose, K. M., Catellier, D., Murphy, C. V., Singh, S., Tegeler, C. H., and Felix, A. (2010). A multicenter evaluation of the ABCD2 score's accuracy for predicting early ischemic stroke in admitted patients with transient ischemic attack. Ann. Emerg. Med. 55, 201-210 e5.

Foulkes, M.A., Wolf, P.A., Price, T. R., Mohr, J. P., and Hier, D. B. (1988). The stroke data bank: design, methods, and baseline characteristics. Stroke 19, 547-554.

Hales, B. M. and Pronovost, P. J. (2006). The checklist--a tool for error management and performance improvement. J. Crit. Care 21, 231-235.

Haynes, A. B., Weiser, T. G., Berry, W. R., Lipsitz, S. R., Breizat, A. H., Dellinger, E. P., Herbosa, T., Joseph, S.,
Kibatala, P. L., Lapitan, M. C., Merry, A. F., Moorthy, K., Reznick, R. K., Taylor, B., and Gawande, A. A. (2009). A surgical safety checklist to reduce morbidity and mortality in a global population. N. Engl. J. Med. 360, 491-499.

Josephson, S. A., Sidney, S., Pham, T. N., Bernstein, A. L., and Johnston, S. C. (2008). Factors associated with the decision to hospitalize patients after transient ischemic attack before publication of prediction rules. Stroke 39, 411-413.

Josephson, S. A., Douglas, V. C., Lawton, M. T., English, J. D., Smith, W.S., and Ko, N. U. (2010). Improvement in intensive care unit outcomes in patients with subarachnoid hemorrhage after initiation of neurointensivist co-management. J. Neurosurg. 112, 626-630.

Kohn, L. T., Janet, C. J., and Donaldson, M. S. (2000). To Err is Human: Building a Safer Health System. Washington, DC: National Academy Press.

Kronick, D.A. (1990). Peer review in 18th-century scientific journalism. JAMA 263, 1321-1322.

Poisson, S. N., Johnston, S. C., and Josephson, S.A. (2010). Urinary tract infections complicating stroke: mechanisms, consequences, and possible solutions. Stroke 41, e180-e184.

Ropper, A. H. (2009). Health care reform and clinical culture. N. Engl. J. Med. 361, e104.

Stelfox, H. T., Palmisani, S., Scurlock, C., Orav, E. J., and Bates, D. W. (2006). The "To Err is Human" report and the patient safety literature. Qual. Saf. Health Care 15, 174-178.

Wachter, R. M. (2010). Patient safety at ten: unmistakable progress, troubling gaps. Health Aff. (Millwood) 29, 165-173.

Received:02 April 2010; accepted:06 April 2010; published online: 17 May 2010.

Citation: Meschia JF (2010) Grand challenge: the emerging field of neurohospitalist medicine. Front. Neur. 1:8. doi: 10.3389/fneur.2010.00008

This article was submitted to Frontiers in Neurocritical and Neurohospitalist Care, a specialty of Frontiers in Neurology. Copyright (C) 2010 Meschia. This is an open-access article subject to an exclusive license agreement between the authors and the Frontiers Research Foundation, which permits unrestricted use, distribution, and reproduction in any medium, provided the original authors and source are credited. 